\title{
Timelike sweeping surfaces according to type-2 Bishop frame in Minkowski 3-Space
}

\author{
Rashad A. Abdel-Baky ${ }^{a}$, Monia F. Naghi ${ }^{b}$ \\ ${ }^{a}$ Department of Mathematics, Faculty of Science, University of Assiut, Assiut 71516, Egypt \\ ${ }^{b}$ Department of Mathematics, Faculty of Science, King Abdulaziz University, Jeddah, Saudi Arabia
}

\begin{abstract}
In this paper, we express timelike sweeping surfaces using rotation minimizing frames in Minkowski3-Space $E_{1}^{3}$.Necessaryandsufficientconditionsfortimelikesweepingsurfacestobedevel-

opable ruled surfaces are derived. Using these, we analyze the conditions when the resulting timelike developable surface is a cylinder, coneortangential surface.
\end{abstract}

Key-Words: - Rotation minimizing frame, Local singularities and convexity.

Received: April 12, 2020. Revised: October 4, 2020. Accepted: October 21, 2020. Published: November 5, 2020.

\section{Introduction}

A sweeping surface is a surface traced by a continuously moving of profile curve along a space curve, which serves as the spine curve. If the spine curve is a straight line, the sweeping surface traced is nothing but a cylindrical surface. If the spine curve is a circular arc, the resulting sweeping surface is a surface of revolution. Consequently, both cylindrical and rotation surfaces can be considered as special sets of sweeping surface [1-8]. A sweeping surface has the ownership that the cross section is a circle and the normal of the circle plane is mostly parallel to that of the cross section. There are several various names for the sweeping surface in previous literature, such as pipe surfaces, tubular surfaces, and canal surfaces.

Several studies in the previous literature that may be inclusived in the area of our treatise are as follows: Do Carmo discussed some geometrical features of pipe surfaces and proved two very remarkable theorems, namely Fary-Milnor theorem and Fenchel's theorem [1]. In [2], Lu and Pottmann proved that a canal surface with a rational spine curve constantly admits a rational parametrization and offered an algorithm for its computation. In [3], Stasiak and Maddocks modeled DNA molecules and parts of proteins as deformable canal surfaces and classified the better packing problem. Maritan et al. [4] studied the problem locating the optimal shapes of closely packed canal surfaces. Caglioti and Giusti offered an automatic reconstruction method to address the reconstruction of a canal surface from a sin- gle image [4]. Krivoshapko, and Hyeng stuided the geometric researches of cyclic surfaces with generating circles of constant and several diameters [5]. Kim et al. [6] considered a particular type of sweeping surface named canal surfaces in Euclidean 3-space. For such a kind of surfaces, some interesting and important relations about the Gaussian curvature, the mean curvature and the second Gaussian curvature are derived. Soliman et al. [7] investigated a particular Weingarten and linear Weingarten varieties of canal surfaces according to Bishop frame in Euclidean 3 -space satisfying some fascinating and necessary equations in terms of the Gaussian curvature, the mean curvature. A significant fact about sweeping surfaces is that they can be developable ruled surfaces [8] . Developable ruled surfaces have a very influential place in mathematics and engineering such as motion analysis or designing cars and ships [9-11]. There are three types of developable ruled surfaces: cylinders, cones and tangent surfaces.

As an useful tool for analyzing curves and surfaces in differential geometry, Serret-Frenet frame is the most familiar and the most used frame field, but not unique, there are also the another frame fields such as Bishop frame or rotation minimizing frame (RMF). Corresponding to Bishop frame in Euclidean space $E^{3}$, there exists a Lorentzian version's frame that is called a Lorentzian Bishop frame, constructed along a curve in Lorentzian space and it is the analog of the Frenet-Serret type frame as applied to Lorentzian geometry. When we discuss a space 
curve, it is more convenient for us to use the Lorentzian Bishop frame along the curve as the basic tool than the Frenet-Serret type frame in Lorentzian space. There are several papers about Lorentzian Bishop frame [12-17].

This manuscript studies the problem to construct a timelike developable surface through a timelike sweeping surface, using a special orthogonal frame of the timelike spine curve, namely the (so. called) rotation minimizing frame (RMF for short). As a result, the necessary and sufficient condition for the timelike sweeping surface to be a timelike developable ruled surface is derived. In particular, we focuses on the study for the resulting timelike developable surface to be cylinder, cone or tangent surface. Finaly, some representative timelike curves are chosen to construct the corresponding timelike developable surfaces which possessing these curves as line of curvatures.

\section{Preliminaries}

Let $R^{3}=\left\{\left(a_{1}, a_{2}, a_{3}\right) \mid, a_{i} \in R(\mathrm{i}=1,2,3)\right\}$ be a 3 -dimensional Cartesian space. For any $\mathbf{a}=\left(a_{1}, a_{2}, a_{3}\right)$, and $\mathbf{b}=\left(b_{1}, b_{2}, b_{3}\right) \in R^{3}$, the pseudo scalar product of $\mathbf{a}$, and $\mathbf{b}$ is specified by $[1,18,19]$ :

$$
<\mathbf{a}, \mathbf{b}>=-a_{1} b_{1}+a_{2} b_{2}+a_{3} b_{3} .
$$

We call $\left(R^{3},<,>\right)$ Minkowski 3-space. We write $E_{1}^{3}$ instead of $\left(R^{3},<,>\right)$. We say that a non-zero vector $\mathbf{a} \in E_{1}^{3}$ is spacelike, lightlike or timelike if $<\mathbf{a}, \mathbf{a}>>0,<\mathbf{a}, \mathbf{a}\rangle=0$ or $<\mathbf{a}, \mathbf{a}><0$ respectively. The norm of the vector $\mathbf{a} \in E_{1}^{3}$ is specified to be $\|\mathbf{a}\|=\sqrt{|<\mathbf{a}, \mathbf{a}\rangle \mid}$. For any two vectors $\mathbf{a}$, $\mathbf{c} \in E_{1}^{3}$, we specify a vector $\mathbf{a} \times \mathbf{c}$ by

$$
\begin{gathered}
\mathbf{a} \times \mathbf{c}\left|\begin{array}{ccc}
-\mathbf{e}_{1} & \mathbf{e}_{2} & \mathbf{e}_{3} \\
a_{1} & a_{2} & a_{3} \\
c_{1} & c_{2} & c_{3}
\end{array}\right| \\
=\left(-\left(a_{2} c_{3}-a_{3} c_{2}\right),\left(a_{3} c_{1}-a_{1} c_{3}\right),\left(a_{1} c_{2}-a_{2} c_{1}\right)\right.
\end{gathered}
$$

where $\mathbf{e}_{1}, \mathbf{e}_{2}, \mathbf{e}_{3}$ is the canonical basis of $E_{1}^{3}$. We can easily check that

$$
\operatorname{det}(\mathbf{a}, \mathbf{c}, \mathbf{b})=<\mathbf{a} \times \mathbf{c}, \mathbf{b}>,
$$

so that $\mathbf{a} \times \mathbf{c}$ is pseudo orthogonal to any $\mathbf{b}=\left(b_{1}, b_{2}, b_{3}\right) \in E_{1}^{3}$.

Let $\beta=\beta(s)$ be a unit speed timelike curve in $E_{1}^{3}$; by $\kappa(s)$ and $\tau(s)$ we indicate the natural curvature and torsion of $\beta=\beta(s)$, respectively. We suppose $\beta^{\prime \prime}(s) \neq 0$ for all $s \in[0, L]$, since this would give us a straight line. Consider the SerretFrenet frame $\{\mathbf{T}(s), \mathbf{N}(s), \mathbf{B}(s)\}$ of $\beta=\beta(s)$, then the Serret-Frenet equations are:

$$
\begin{aligned}
\left(\begin{array}{l}
\mathbf{T}^{\prime} \\
\mathbf{N}^{\prime} \\
\mathbf{B}^{\prime}
\end{array}\right)=\left(\begin{array}{lll}
0 & \kappa(s) & 0 \\
\kappa(s) & 0 & \tau(s) \\
0 & -\tau(s) & 0
\end{array}\right)\left(\begin{array}{l}
\mathbf{T} \\
\mathbf{N} \\
\mathbf{B}
\end{array}\right) \\
=\omega \times\left(\begin{array}{l}
\mathbf{T} \\
\mathbf{N} \\
\mathbf{B}
\end{array}\right)
\end{aligned}
$$

where $\omega(s)=\tau \mathbf{T}+\kappa \mathbf{B}$ is Darboux vector of the Serret-Frenet frame. In this paper, dash indicate the derivatives with respect to arc-length parameter $s$. It is easy to see that

$$
\mathbf{T} \times \mathbf{N}=\mathbf{B}, \mathbf{T} \times \mathbf{B}=-\mathbf{N}, \mathbf{N} \times \mathbf{B}=-\mathbf{T} .
$$

Definition 2.1. A pseudo orthogonal moving frame $\left\{\xi_{1}, \xi_{3}, \xi_{3}\right\}$, along a non null space curve $\alpha(s)$, is rotation minimizing frame (RMF) with respect to $\xi_{1}$ if the derivatives of $\xi_{2}$ and $\xi_{3}$ are both parallel to $\xi_{1}$, or its angular velocity $\omega$ satisfies $<\omega, \xi_{1}>=0$. A similar characterization holds when $\xi_{2}$ or $\xi_{3}$ is chosen as the reference direction.

According to the Definition 2.1, we observe that the Serret-Frenet frame is RMF with respect to the principal normal $\mathbf{N}$, but not with respect to the tangent $\mathbf{T}$ and the binormal $\mathbf{B}$. Although the Serret-Frenet frame is not RMF with respect to $\mathbf{T}$, one can easily obtain such a RMF from it. New normal plane vectors $\left(\mathbf{N}_{1}, \mathbf{N}_{2}\right)$ are identify through a rotation of $(\mathbf{N}, \mathbf{B})$ according to

$$
\left(\begin{array}{l}
\mathbf{T}_{1} \\
\mathbf{N}_{1} \\
\mathbf{N}_{2}
\end{array}\right)=\left(\begin{array}{lll}
1 & 0 & 0 \\
0 & \cos \vartheta & \sin \vartheta \\
0 & -\sin \vartheta & \cos \vartheta
\end{array}\right)\left(\begin{array}{l}
\mathbf{T} \\
\mathbf{N} \\
\mathbf{B}
\end{array}\right)
$$

with a certain spacelike angle $\vartheta(s) \geq 0$. Here, we will call the set $\left\{\mathbf{T}_{1}, \mathbf{N}_{1}, \mathbf{N}_{2}\right\}$ as RMF or Bishop frame. The RMF vector satisfy the relations

$\mathbf{T}_{1} \times \mathbf{N}_{1}=\mathbf{N}_{2}, \mathbf{T}_{1} \times \mathbf{N}_{2}=-\mathbf{N}_{1}, \mathbf{N}_{1} \times \mathbf{N}_{2}=-\mathbf{T}_{1}$.

As a result, we have the alternative frame equations

$$
\begin{gathered}
\left(\begin{array}{c}
\mathbf{T}_{1}^{\prime} \\
\mathbf{N}_{1}^{\prime} \\
\mathbf{N}_{2}^{\prime}
\end{array}\right)=\left(\begin{array}{ccc}
0 & \kappa_{1}(s) & -\kappa_{2}(s) \\
\kappa_{1}(s) & 0 & 0 \\
-\kappa_{2}(s) & 0 & 0
\end{array}\right)\left(\begin{array}{l}
\mathbf{T}_{1} \\
\mathbf{N}_{1} \\
\mathbf{N}_{2}
\end{array}\right) \\
=\widetilde{\omega} \times\left(\begin{array}{l}
\mathbf{T}_{1} \\
\mathbf{N}_{1} \\
\mathbf{N}_{2}
\end{array}\right),
\end{gathered}
$$


where $\widetilde{\omega}(s)=\kappa_{2} \mathbf{N}_{1}+\kappa_{1} \mathbf{N}_{2}$ is RMF Darboux vector. Here, the Bishop curvatures are defined by $\kappa_{1}(s)=\kappa \cos \vartheta$, and $\kappa_{2}(s)=\kappa \sin \vartheta$. One can show that

$$
\left.\begin{array}{l}
\kappa(s)=\sqrt{\kappa_{1}^{2}+\kappa_{2}^{2}}, \text { and } \vartheta=\tan ^{-1}\left(\frac{\kappa_{2}}{\kappa_{1}}\right) ; \kappa_{1} \neq 0, \\
\vartheta(s)=\vartheta_{0}-0 s \int \tau d s, \vartheta_{0}=\vartheta(0) .
\end{array}\right\}
$$

Comparing Eq. (4) with Eq. (8) we pay attention that the relative velocity is

$$
\widetilde{\omega}(s)-\omega(s)=\tau \mathbf{T} .
$$

As a consequence, the Serre-Frenet frame and the RMF are identical if and only if $\beta(s)$ is a planar, i.e. $\tau(s)=0$.

We symbolize a surface $M$ in $E_{1}^{3}$ by

$$
\begin{aligned}
M: \mathbf{X}(s, u)= & \left(x_{1}(s, u), x_{2}(s, u), x_{3}(s, u),\right. \\
& (s, u) \in D \subseteq R^{2} .
\end{aligned}
$$

Let $\mathbf{U}$ be the standard unit normal vector field on a surface $M$ located by $\mathbf{U}=\frac{\mathbf{X}_{u} \times \mathbf{X}_{s}}{\left\|\mathbf{X}_{u} \times \mathbf{X}_{s}\right\|}$, where, $\mathbf{X}_{i}=\frac{\partial \mathbf{X}}{\partial i}$. Then the metric (first fundamental form) $I$ of a surface $M$ is

$$
I=g_{11} d s^{2}+2 g_{12} d s d u+g_{22} d u^{2},
$$

where $g_{11}=<\mathbf{X}_{s}, \mathbf{X}_{s}>, g_{12}=<\mathbf{X}_{u}, \mathbf{X}_{s}>$ , $g_{22}=<\mathbf{X}_{u}, \mathbf{X}_{u}>$. We define the second fundamental form $I I$ of $M$ by

$$
I I=h_{11} d s^{2}+2 h_{12} d s d u+h_{22} d u^{2},
$$

where $h_{11}=<\mathbf{X}_{s s}, \mathbf{U}>, h_{12}=<\mathbf{X}_{u s}, \mathbf{u}>$ , $h_{22}=<\mathbf{X}_{u u}, \mathbf{U}>. \quad M$ is called a spacelike/timelike surface if the induced metric is Riemannian/Lorentzian metric on each tangent plane. Therefore, since $M$ is timelike and spacelike, respectively, we have

$$
\begin{gathered}
\operatorname{det}\left(g_{i j}\right)=g_{11} g_{22}-g_{12}^{2}<0, \\
\text { and } \operatorname{det}\left(g_{i j}\right)=g_{11} g_{22}-g_{12}^{2}>0 .
\end{gathered}
$$

The Gaussian curvature $K$ is

$K(s, u)=\epsilon \frac{h_{11} h_{22}-h_{12}^{2}}{g_{11} g_{22}-g_{12}^{2}}$, with $\epsilon=<\mathbf{U}, \mathbf{U}>= \pm 1$.

\section{Timelike sweeping surface}

The concept of a sweeping surface is defined kinematically by a plane curve moving through space such that the movement of any point on the surface is always orthogonal to the plane. By utilizing the type-2 Bishop frame, the timelike sweeping surface family which pass through the timelike curve $\beta(s)$ is described by

$$
\begin{aligned}
& M: \mathbf{X}(s, u)=\beta(s)+A(s) \mathbf{x}(u) \\
& =\beta(s)+x_{1}(u) \mathbf{N}_{1}+x_{2}(u) \mathbf{N}_{2},
\end{aligned}
$$

where the profile curve $\mathbf{x}(u)=\left(0, x_{1}(u), x_{2}(u)\right)^{t}$, the symbol ' $t$ ' represents transposition, with another parameter $0 \leq u \leq U$. The semi orthogonal matrix $A(s)=\left\{\mathbf{T}(s), \mathbf{N}_{1}(s), \mathbf{N}_{2}(s)\right\}$ specifies the RMF along $\beta(s)$. Geometrically, the sweeping surface $\mathbf{X}(s, u)$ is generated by moving the profile curve $\mathbf{x}(u)$ along the spine curve $\beta(s)$ with the orientation as specified by $A(s)$. Profile curve $\mathbf{x}(u)$ is in the $2 \mathrm{D}$ or $3 \mathrm{D}$ space which passes through the spine curve $\beta(s)$ during sweeping. Clearly, the sweeping technique leaves the designer with one degree of freedom, as it is still possible to revolve the RMF around. We now analyze the relation between the differentiability of the timelike spine curve and of the identical sweeping surface. Next, without loss of generality, we can suppose that the profile curve $\mathbf{x}(u)$ is a unit speed spacelike curve, i.e. $. x_{1}^{2}+. x_{2}^{2}=1$. Also, we use "dot" to denote the derivative with respect to the arc length parameter of the profile curve $\mathbf{x}(u)$. Then, the tangent vectors and the unit normal vector to the surface, respectively, are

$$
\left.\begin{array}{l}
\mathbf{X}_{s}(s, u)=\left(1+\kappa_{1} x_{1}-\kappa_{2} x_{2}\right) \mathbf{T}_{1}, \\
\mathbf{X}_{u}(s, u)=. x_{1} \mathbf{N}_{1}+. x_{2} \mathbf{N}_{2},
\end{array}\right\}
$$

The unit normal vector of $M$ is

$$
\mathbf{U}(s, u):=\frac{\mathbf{X}_{u} \times \mathbf{X}_{s}}{\left\|\mathbf{X}_{u} \times \mathbf{X}_{s}\right\|}=-. x_{2} \mathbf{N}_{1}+. x_{1} \mathbf{N}_{2} .
$$

Note that $\|\mathbf{U}(s, u)\|^{2}=1$ means that $M$ is a timelike surface.

Proposition 3.1. Consider a point in the normal plane of the spine timelike curve $\beta(s)$. The tangent vector of its trajectory $\beta(s)+A(s) \mathbf{x}(u)$, that is generated by the RMF, is always parallel to the tangent vector of $\beta(s)$.

By simple calculations, we have the following:

$$
\left.\begin{array}{l}
g_{11}=-\left(1+\kappa_{1} x_{1}-\kappa_{2} x_{2}\right)^{2} \\
g_{12}=0, g_{22}=1
\end{array}\right\}
$$


Furthermore, we have:

$$
\left.\begin{array}{l}
\mathbf{X}_{s s}=\left(\kappa_{1}^{\prime} x_{1}-\kappa_{2}^{\prime} x_{2}\right) \mathbf{T}_{1} \\
\quad+\left(1+\kappa_{1} x_{1}-\kappa_{2} x_{2}\right)\left(\kappa_{1} \mathbf{N}_{1}-\kappa_{2} \mathbf{N}_{2}\right), \\
\mathbf{X}_{s u}=\left(\kappa_{1} \cdot x_{1}+\kappa_{2} \cdot x_{2}\right) \mathbf{T}_{1}, \\
\mathbf{X}_{u u}=. . x_{1} \mathbf{N}_{1}+. . x_{2} \mathbf{N}_{2} .
\end{array}\right\}
$$

Thus, we arrive by means of Eqs. (18), and (20), at

$$
\left.\begin{array}{l}
h_{11}=-\left(1+\kappa_{1} x_{1}-\kappa_{2} x_{2}\right)\left(\kappa_{1} . x_{2}+\kappa_{2} . x_{1}\right), \\
h_{12}=0, h_{22}=. x_{1} . . x_{2}-. x_{2} . . x_{1} .
\end{array}\right\}
$$

Hence, the $u$-and $s$ curves of $M$ are lines of curvature, that is, $g_{12}=h_{12}=0$. So, the isoparametric curve

$$
\begin{aligned}
\pi(u): \zeta(u):= & \mathbf{X}\left(u, s_{0}\right)=\beta\left(s_{0}\right)+x_{1}(u) \mathbf{N}_{1}\left(s_{0}\right) \\
& +x_{2}(u) \mathbf{N}_{2}\left(s_{0}\right),
\end{aligned}
$$

is a planar unit speed spacelike line of curvature. Eq. (22) define a set of oneparameter family of timelike planes in $E_{1}^{3}$. The spacelike unit tangent vector to $\zeta(u)$ is $\mathrm{T}_{\zeta}(u)=. x_{1} \mathbf{N}_{1}\left(s_{0}\right)+. x_{2} \mathbf{N}_{2}\left(s_{0}\right)$, and thus the spacelike unit principal normal vector of $\zeta(u)$ is given by $\mathrm{N}_{\zeta}(u)=\mathbf{T}_{1}\left(s_{0}\right) \times \mathbf{T}_{\zeta}(u)=$ $-. x_{2} \mathbf{N}_{1}+. x_{1} \mathbf{N}_{2}=\mathbf{U}\left(s_{0}, u\right)$.

Consequently, the surface spacelike normal $\mathbf{U}\left(s_{0}, u\right)$ is identical with the spacelike principal normal $\mathbf{N}_{\zeta}(u)$, i.e., the curve $\zeta(u)$ is a geodesic planar spacelike line of curvature on $\mathbf{X}\left(u, s_{0}\right)$. Surfaces whose parametric curves are lines of curvature have various applications in geometric design [6-8]. In the case of sweeping surfaces, one has to compute the offset surfaces $\mathbf{X}_{f}(u, s)=\mathbf{X}(u, s)+f \mathbf{U}(s, u)$ of a given surface $\mathbf{X}(u, s)$ at a certain distance $f$. In consequence of this equation, the offsetting process for timelike sweeping surface can be reduced to the offsetting of planar profile spacelike curve, which is very easier to deal with. Hence, we can state the following proposition:

Proposition 3.2. Consider a timelike sweeping surface $M$ represented by Eq. (16). Let $\mathbf{x}_{f}(u)$ be the spacelike planar offset of the profile curve $\mathbf{x}(u)$ at distance $f$. Then the offset timelike surface $\mathbf{Q}_{f}(u, s)$ is again a timelike sweeping surface, generated by the timelike spine curve $\beta(s)$ and profile spacelike curve $\mathbf{x}_{f}(u)$.

\subsection{Local singularities and convexity}

Singularities and convexity are essential for understanding the properties of sweeping surfaces and are investigated in the following: A point of the timelike surface $M$ is called singular if and only if the first derivatives are linearly dependent. Owing to Proposition 3.1, this is only possible if $(\partial / \partial s) \mathbf{X}(s, u)=\mathbf{0}$. Thus, we obtain the following condition:

$$
\left\|\mathbf{X}_{u} \times \mathbf{X}_{s}\right\|=1+\kappa_{1} x_{1}-\kappa_{2} x_{2}=0
$$

which leads to

$$
\rho+x_{1} \cos \vartheta-x_{2} \sin \vartheta=0,
$$

where $\rho(s)=\kappa^{-1}$ is the radius of curvature of $\beta(s)$. Using $\rho(s)$, we have the relations

$$
x_{1}=-\rho \cos \vartheta, x_{2}=\rho \sin \vartheta,
$$

where $\vartheta=\vartheta(s)$. In consequence of its kinematical generation, the sweeping surface $\mathbf{X}(s, u)$ has singular points exactly at intersections of the spacelike profile curve $\mathbf{x}=\mathbf{x}(u)$ and the spacelike curvature axis (rotation axis),

$$
L(u)=\left\{\left(x_{1}, x_{2}\right) \mid \rho+x_{1} \cos \vartheta-x_{2} \sin \vartheta=0\right\} .
$$

Thus, the timelike sweeping surface has a second order contact with the timelike surface of revolution that is generated by simply rotating the profile spacelike curve $\mathbf{x}=\mathbf{x}(u)$ around spacelike axis $L(u)$. Hence, we obtain the following corollary:

Corollary 3.1. The timelike sweeping surface $M$ represented by Eq. (16), has no singular points if the condition

$$
\rho+x_{1} \cos \vartheta-x_{2} \sin \vartheta \neq 0,
$$

is satisfied for all $s$, and $u$.

In order to study the shape of $M$ we investigate the Gaussian curvature $K(s, u)=\chi_{1} \chi_{2}$. Here, the $\chi_{i}(s, u)(i=1,2)$ are the principal curvatures. From Eqs. (6), and (18), we obtain

$$
\begin{gathered}
\mathbf{U}(s, u)=-\left(. x_{2} \cos \vartheta+. x_{1} \sin \vartheta\right) \mathbf{N} \\
+\left(-. x_{2} \sin \vartheta+. x_{1} \cos \vartheta\right) \mathbf{B} .
\end{gathered}
$$

Further, since $g_{12}=h_{12}=0$, the principal curvature $\chi_{1}\left(s_{0}, u\right)$ of the profile curve $\mathbf{x}(u)$ is

$$
\chi_{1}:=\|. \mathbf{x} \times . . \mathbf{x}\|\|\cdot \mathbf{x}\|^{-3}=. x_{1} . . x_{2}-. x_{2} . . x_{1} .
$$

The curvature of the $s$-parameter curve $(u$ constant) is

$$
\chi\left(s, u_{0}\right):=\frac{\left\|\mathbf{X}_{s} \times \mathbf{X}_{s s}\right\|}{\left\|\mathbf{X}_{s}\right\|^{3}}=\frac{1}{\rho+x_{1} \cos \vartheta-x_{2} \sin \vartheta} .
$$


The principal curvature $\chi_{2}$ is related to the curvature $\chi(s, u)$ via Meusnier's Theorem [1]:

$$
\chi_{2}=\chi(s, u) \cos \varphi
$$

where $\varphi$ is spacelike angle of $\mathbf{U}$, and $\mathbf{N}$. Thus, the Gaussian curvature $K(s, u)$ can be defined by

$$
K(s, u)=\chi_{1} \chi(s, u) \cos \varphi .
$$

In CAGD, conditions that guarantee the convexity or curves which output parabolic points of a surface are required in different applications [6-9]. However, for the timelike sweeping surface $M$ the convexity can be controlled with the help of the Gaussian curvature as:

$$
K(s, u)=0 \Leftrightarrow \chi_{1} \chi(s, u) \cos \varphi=0 .
$$

It can be seen that there are three cases which make parabolic points:

Case (1) exists when $\chi_{1}=0$. If $\chi_{1}=0$, the profile curve $\mathbf{x}=\mathbf{x}(u)$ is degenerate into a spacelike straight line, from Eq. (24), it can be seen that

$$
\chi_{1}=0 \Leftrightarrow . \mathbf{x} \times . . \mathbf{x}=\mathbf{0} \Leftrightarrow . \mathbf{x} \| . . \mathbf{x} .
$$

This equation shows that an inflection or flat point of the profile spacelike curve generates a parabolic curve $s=$ const. on parts of $M$.

Case (2) exists when $\chi(s, u)=0$. Thus, an infection or flat point of the spine curve generates an isoparametric parabolic curve $\mathrm{u}=$ const. on $M$.

Case (3) exists when $\varphi=\pi / 2$. Owing to Eqs. (23), and (26), these parabolic curves are characterized by the following condition

$$
. x_{2} \cos \vartheta+. x_{1} \sin \vartheta=0,
$$

is satisfied for all $s$, and $u$. In this case, the spine timelike curve $\beta$ is not only a line of curvature but as well a geodesic spacelike curve on $M$.

In $\mathrm{CAD}$, conditions that surety the convexity of a surface are required in various applications (such as manufacturing of sculptured surfaces, or layered manufacturing). In the case of the timelike sweeping surface $M$, however, the convexity can be planned with the help of the differential geometric properties, as follows: By integration of Eq. (29), the following equation can be obtained

$$
x_{2} \sin \vartheta+x_{1} \cos \vartheta=h(s),
$$

where $h=h(s)$ is an arbitrary function. Then we have the relations

$$
x_{1}=h(s) \cos \vartheta, x_{2}=h(s) \sin \vartheta .
$$

When Eqs. (30) are applied to Eq. (16), with attention of Eq. (6), we instantly find that the expression of the parabolic curve is

$$
\gamma(s)=\beta(s)+h(s) \mathbf{B}(s) .
$$

From the above analysis the following conclusions can be attain.

Corollary 3.2. Let $M$ be a timelike sweeping surface expressed by Eq. (16), with timelike spine curve, and spacelike profile curve have non-vanishing curvatures everywhere. Then $M$ has exactly one parabolic line if and only if the spine curve is a spacelike geodesic curve.

\subsection{Timelike developable ruled surfaces}

In this subsection, we analyze the case, that the profile curve $\mathbf{x}(u)$ degenerates to a spacelike straight line. If $\mathbf{x}(u)=(0,0, u)^{t}$, we have the following timelike developable surface

$$
F: \mathbf{Q}(s, u)=\beta(s)+u \mathbf{N}_{2}(s), u \in R .
$$

Similarly, we have the following timelike developable surface

$$
F^{\perp}: \mathbf{Q}^{\perp}(s, u)=\beta(s)+u \mathbf{N}_{1}(s), u \in R .
$$

Proposition 3.3. Let $M$ be a timelike sweeping surface expressed by Eq. (3.1), if the profile curve $\mathbf{x}(u)$ degenerates to a spacelike straight line, then $M$ is a timelike developable surface.

Furthermore, it is possible to show $\mathbf{Q}(s, 0)=$ $\beta(s)\left(\operatorname{resp} . \mathbf{Q}^{\perp}(s, 0)=\beta(s)\right), 0 \leq s \leq L$, that is, the surface $F$ (resp. $F^{\perp}$ ) interpolates the curve $\beta(s)$. Furthermore, since

$$
\mathbf{Q}_{s} \times \mathbf{Q}_{u}:=-\left(1-u \kappa_{2}\right) \mathbf{N}_{1}(s),
$$

then $F^{\perp}$ is the normal timelike developable surface of $F$ along $\beta(s)$. Therefore, the timelike surface $F$ (resp. $F^{\perp}$ ) interpolates the timelike curve $\beta(s)$, and $\beta(s)$ is a timelike line of curvature of $F$ (resp. $F^{\perp}$ ). Hence, we can classify the singularities of developable surface $F$ by using $\kappa_{2}$.

Theorem 3.1. (Existence and uniqueness). Under the above notations there exists a unique timelike developable surface expressed by Eq. (32).

Proof. For the existence, we have the timelike developable surface expressed by Eq. (32). On 
the other hand, since $F$ is a ruled surface, we assume that

$$
\left.\begin{array}{l}
F: \mathbf{Q}(s, u)=\beta(s)+u \mathbf{a}(s), u \in R, \\
\mathbf{a}(s)=a_{1}(s) \mathbf{T}_{1}+a_{2}(s) \mathbf{N}_{1}+a_{3}(s) \mathbf{N}_{2}, \\
\|\mathbf{a}(s)\|^{2}=-a_{1}^{2}+a_{2}^{2}+a_{3}^{2}=1, \mathbf{a}^{\prime}(s) \neq \mathbf{0} .
\end{array}\right\}
$$

It can be immediately seen that $F$ is developable if and only if

$\operatorname{det}\left(\beta^{\prime}, \mathbf{a}, \mathbf{a}^{\prime}\right)=0 \Leftrightarrow a_{2}\left(a_{3}^{\prime}-a_{1} \kappa_{2}\right)-a_{3}\left(a_{2}^{\prime}+a_{1} \kappa_{1}\right)=0$

On the other hand, in view of Eq. (34), we have

$$
\left(\mathbf{Q}_{s} \times \mathbf{Q}_{u}\right)(s, u)= \pm \lambda(s, u) \mathbf{N}_{1},
$$

where $\lambda=\lambda(s, u)$ is a differentiable function. Further, the normal vector $\mathbf{Q}_{s} \times \mathbf{Q}_{v}$ at the point $(s, 0)$ is

$$
\left(\mathbf{Q}_{s} \times \mathbf{Q}_{u}\right)(s, 0)=-a_{3} \mathbf{N}_{1}+a_{2} \mathbf{N}_{2}
$$

Thus, from Eqs. (37), and (38), one finds that:

$$
a_{2}=0, \operatorname{and}_{3}=\lambda(s, 0),
$$

which follows from Eq. (3.23) that $a_{3} a_{1} \kappa_{1}=0$, which leads to $a_{1} a_{3}=0$, with $\kappa_{1} \neq 0$. If $(s, 0)$ is a regular point (i.e., $\lambda(s, 0) \neq 0$ ), then $a_{3}(s) \neq 0$, and $a_{1}=0$. Therefore, the direction of $\mathbf{a}(s)$ is the direction of $\mathbf{N}_{2}(s)$. This means that uniqueness holds .

In analogous arguments for $F^{\perp}$, we can give the corresponding Theorem of Theorem 3.1. we omit the details here. Thus, the Joachimsthal theorem can be stated as the following:

Theorem 3.2. (Joachimsthal). Let $F$ and $F^{\perp}$ two timelike developable surfaces such that $F \cap F^{\perp}=\beta(s)$ is a timelike regular curve and $<\mathbf{N}_{1}, \mathbf{N}_{2}>=0$ along $\beta(s)$, where $\mathbf{N}_{1}$ and $\mathbf{N}_{2}$ are unitary spacelike normal vector fields to $F$ and $F^{\perp}$, respectively. Then $\beta(s)$ is a timelike line of curvature of $F$ if and only if it is a timelike line of curvature of $F^{\perp}$.

In above theorem, we not only prove the existence and uniqueness of the timelike developable surface, but also give the exact term of the surface. This is very significance full in practical application. As is will known, there are three kinds of developable surfaces, the given curve can be classified into three kinds correspondingly. In what follows, we will discuss the relationship among the given timelike curve $\beta(s)$ and its isoparametric timelike developable. The first case is when,

$$
\mathbf{N}_{2} \times \mathbf{N}_{2}^{\prime}=\mathbf{0} \Leftrightarrow \kappa \sin \vartheta \mathbf{N}_{1}=\mathbf{0} .
$$

In this case, $F$ is referred to as a cylindrical surface. Since $\mathbf{N}_{1} \neq \mathbf{0}$, then $F$ is a timelike cylindrical surface if and only if $\sin \vartheta=0 \Leftrightarrow \vartheta=0$ or $\pi$. However, in any case, we have $\vartheta^{\prime}=0$, then $\tau=0$. That is, the curve $\beta$ is a timelike planar curve, and $F$ is a timelike binormal surface. As a result the following theory can be given:

Theorem 3.3. The timelike developable surface Eq. (32) is a cylinder surface if and only if $\vartheta=0$ or $\pi$.

On the other hand, likwise, we can also have the following:

$$
\mathbf{N}_{2} \times \mathbf{N}_{2}^{\prime} \neq \mathbf{0} .
$$

This implies that $F$ is a non-cylindrical surface. Therefore, the first derivative of the directrix is

$$
\beta^{\prime}(s)=\mathbf{C}^{\prime}(s)+\sigma(s) \mathbf{N}_{2}^{\prime}(s)+\sigma^{\prime}(s) \mathbf{N}_{2}(s),
$$

where $\mathbf{C}^{\prime}$ is the first derivative of the striction curve, $\sigma(s)$ is a smooth function. Therefore, we obtain

$$
<\mathbf{N}_{2} \times \mathbf{N}_{2}^{\prime}, \mathbf{C}^{\prime}>=0 .
$$

Similarly, there are two possible cases which satisfy Eq. (43), as in the following: The first one is when the first derivative of the striction curve is $\mathbf{C}^{\prime}=0$. Geometrically this condition implies that the striction curve degenerates to a constant point, and the ruled surface becomes a timelike cone; the striction point of a cone is its vertex. Thus, the surface $F$ is a timelike cone if and only if there exists a fixed point $\mathbf{C}$ and a function $\sigma(s)$ such that $\sigma \kappa \sin \vartheta=1, \sigma^{\prime}=0$, which imply that

$$
\sigma=\text { const. }=\frac{1}{\kappa \sin \vartheta} \Leftrightarrow \kappa \sin \vartheta=\kappa_{0} \sin \vartheta_{0},
$$

where $\vartheta_{0}=\vartheta(0)$, and $\kappa_{0}=\kappa(0)$. In Eq. (44), $\kappa \sin \vartheta$ is a constant. However, if $\vartheta$ is constant, then $\tau=0$, that is, $\beta(s)$ is a timelike planar line of curvature, and $\kappa$ is also constant. Also, if $\kappa$ is a constant, we can have $\tau=0$, and $\vartheta$ is a constant. Then the curve $\beta(s)$ is the arc of a Lorentzian circle.

Theorem 3.4. The timelike developable surface Eq. (32) is a cone if and only if $\kappa \sin \vartheta=\kappa_{0} \sin \vartheta_{0}$, where $\vartheta_{0}=\vartheta(0)$, and $\kappa_{0}=\kappa(0)$. 
Theorem 3.5. The timelike developable surface Eq. (32) is a tangential surface if and only if $\kappa \sin \vartheta \neq \kappa_{0} \sin \vartheta_{0}$, where $\vartheta_{0}=\vartheta(0)$, and $\kappa_{0}=\kappa(0)$.

Proof. According to the proof of Theorem 3.4, if $\kappa \sin \vartheta \neq \kappa_{0} \sin \vartheta_{0}$, then $\mathbf{C}^{\prime} \neq \mathbf{0}$. Since ${ }_{i} \mathbf{N}_{2} \times \mathbf{N}_{2}^{\prime}, \mathbf{C}^{\prime}>=0$, and $<\mathbf{N}_{2}, \mathbf{N}_{2}^{\prime}>=0$, and the condition for $\mathbf{C}$ to be striction curve is equivalent to $\left\langle\mathbf{C}^{\prime}, \mathbf{N}_{2}\right\rangle=0$ we can get $\mathbf{C}^{\prime} \| \mathbf{N}_{2}$. This means the tangent surface is composed of tangents of a timelike spatial curve, the cuspidal edge $\beta(s)$.

\subsection{Examples}

$\mathrm{n}$ what follows, we will discuss the construction of timelike developable surfaces with the given curve as a line of curvature.

Example 1. Given the timelike circle

$$
\beta(s)=(\sinh s, 0, \cosh s), \quad-1 \leq s \leq 1 .
$$

By computing, we get

$$
\begin{aligned}
& \mathbf{T}(s)=(\cosh s, 0, \sinh s) \\
& \mathbf{N}(s)=(\sinh s, 0, \cosh s) \\
& \mathbf{B}(s)=(0,-1,0) \\
& \kappa=1, \tau=0
\end{aligned}
$$

Now, we will obtain the $\operatorname{RMF}\left\{\mathbf{T}_{1}(s), \mathbf{N}_{1}(s)\right.$, $\left.\mathbf{N}_{2}(s)\right\}$ as follows: From $\tau(s)=0$, we find $\vartheta(s)=\vartheta_{0}$ is a constant. Therefore, the transformation matrix can be expressed as:

$$
\left(\begin{array}{l}
\mathbf{T}_{1} \\
\mathbf{N}_{1} \\
\mathbf{N}_{2}
\end{array}\right)=\left(\begin{array}{lll}
1 & 0 & 0 \\
0 & \cos \vartheta_{0} & \sin \vartheta_{0} \\
0 & -\sin \vartheta_{0} & \cos \vartheta_{0}
\end{array}\right)\left(\begin{array}{l}
\mathbf{T} \\
\mathbf{N} \\
\mathbf{B}
\end{array}\right),
$$

From this, we have

$$
\begin{aligned}
& \mathbf{N}_{1}=\left(\cos \vartheta_{0} \sinh s,-\sin \vartheta_{0}, \cos \vartheta_{0} \cosh s\right), \\
& \mathbf{N}_{2}=\left(-\sin \vartheta_{0} \sinh s,-\cos \vartheta_{0}, \sin \vartheta_{0} \cosh s\right)
\end{aligned}
$$

Let $x_{1}(u)=\cos u, x_{2}(u)=\sin u$, and $-\pi \leq u \leq$ $\pi$, the corresponding timelike sweeping surface can be expressed as

$M: \mathbf{X}(s, u)=(\cosh s, 0, \sinh s)+\cos u \mathbf{N}_{1}+\sin u \mathbf{N}_{2}$.

The timelike developable surface $F$ is

$\mathrm{F}: \mathrm{Q}(\mathrm{s}, \mathrm{u})=(\cosh s, 0, \sinh s)$

$+u\left(-\sin \vartheta_{0} \sinh s,-\cos \vartheta_{0}, \sin \vartheta_{0} \cosh s\right)$.

1) If $\vartheta_{0}=0$ or $\pi$, obviously, according to Theorem 3.3, the developable surface is a timelike cylinder (see Fig. 1). In this case, the graph of the corresponding timelike sweeping surface is shown in Fig. 2.

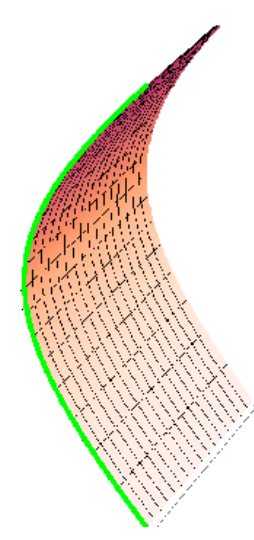

Figure 1: The timelike cylinder with $\vartheta_{0}=0$.

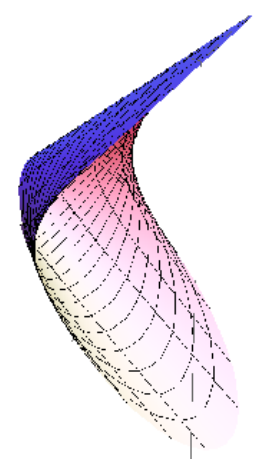

Figure 2: The timelike sweeping surface with $\vartheta_{0}=0$

2) Choosing $\vartheta_{0}=\frac{\pi}{4}$, obviously, according to Theorem 3.4, the corresponding surface is a timelike cone as shown in Fig. 3. Simillarly, the corresponding timelike sweeping surface is shown in Fig. 4.

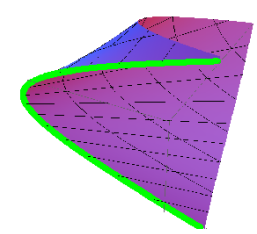

Figure 3: The timelike cone with $\vartheta_{0}=\frac{\pi}{4}$.

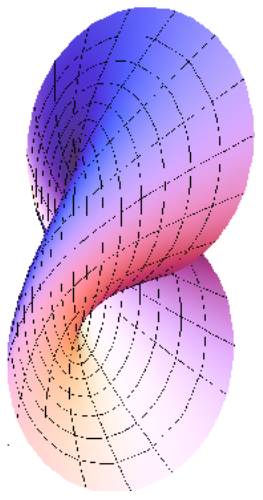

Figure 4: The timelike sweeping surface with $\vartheta_{0}=\frac{\pi}{4}$ 
Example 2. Given the timelike cylindrical helix

$$
\beta(s)=(\sqrt{2} \sinh s, s, \sqrt{2} \cosh s),-1 \leq s \leq 1 .
$$

The Serret-Frenet frame can be computed as follows

$$
\left.\begin{array}{l}
\mathbf{T}(s)=(\sqrt{2} \cosh s, 1, \sqrt{2} \sinh s), \\
\mathbf{N}(s)=(\sinh s, 0, \cosh s), \\
\mathbf{B}(s)=(-\cosh s,-\sqrt{2},-\sinh s), \\
\kappa=\sqrt{2}, \tau=-1 .
\end{array}\right\}
$$

By a similar procedure as in Example (1), we have $\vartheta(s)=s+\vartheta_{0}$. Then we would obtain

$$
\begin{aligned}
& \mathbf{N}_{1}=\left(\begin{array}{l}
N_{11} \\
N_{12} \\
N_{13}
\end{array}\right)=\left(\begin{array}{l}
\cos \vartheta \sinh s-\sin \vartheta \cosh s \\
-\sqrt{2} \sin \vartheta \\
\cos \vartheta \cosh s-\sin \vartheta \sinh s
\end{array}\right), \\
& \mathbf{N}_{2}=\left(\begin{array}{l}
N_{21} \\
N_{22} \\
N_{23}
\end{array}\right)=\left(\begin{array}{l}
-\sin \vartheta \sinh s-\cos \vartheta \cosh s \\
-\sqrt{2} \cos \vartheta \\
-\sin \vartheta \cosh s-\cos \vartheta \sinh s
\end{array}\right) .
\end{aligned}
$$

Figure 8: The timelike sweeping surface with $\vartheta_{0}=$ 0.5

\section{Conclusion}

We discuss some aspects of the differential geometry of timelike sweeping surface by setting up an orthonormal RMF to each point of the spine timelike curve. Then, we have solve the problem of requiring the timelike surface that is timelike sweeping surface and at the same time timelike developable surface. This study is intended to clear away to conduct the geometric analysis of timelike developable ruled surfaces through the timelike sweeping surfaces. An analogue of the problem addressed in this paper may be consider in the Galilean 3-space $\mathrm{G}^{3}$.

\section{References:}

According to Theorem 3.5, the timelike developable surface

$$
\begin{aligned}
F: & \mathbf{Q}(s, u)=\beta(s)+u \mathbf{N}_{2} \\
= & \left(\begin{array}{l}
\sqrt{2} \sinh s-u(\sin \vartheta \sinh s+\cos \vartheta \cosh s) \\
s-u \sqrt{2} \cos \vartheta \\
\sqrt{2} \cosh s-u(\sin \vartheta \sinh s+\cos \vartheta \cosh s)
\end{array}\right)
\end{aligned}
$$

is a tangent surface. The graphs of the timelike $M$, and $F$ surfaces are shown in Figs. 6,$7 ; \vartheta_{0}=$ 0 . Figures 7,8 shows the corresponding timelike $M$, and $F$ surfaces with $\theta_{0}=0.5$.
[1] MP. do Carmo. Differential Geometry of Curves and Surface, Prentice-Hall, Englewood Cliffs, NJ, 1976.

[2] W. Lu, and H. Pottmann. Pipe surfaces with rational spine curve are rational, Comput. Aided Geom. Des., 13 (1996), pp. 621-628.

[3] A. Stasiak, JH. Maddocks. Best Packing in Proteins and DNA, Nature London , 406 (2000), pp. 251-253.

[4] A. Maritan, C. Micheletti, A. Trovato, J.R Banavar. Optimal shapes of compact strings," Nature London , 406 (2000), pp. 287-290. 
[5] S.N. Krivoshapko, and C.A. Hyeng. Classi cation of cyclic surfaces and geometrical research of canal surfaces, Int. J. Res. Rev. Appl. Sci. 12 (2012), No. 3, PP. 360-374.

[6] Y.H Kim, H. Liu, and J. Qian. Some Characterizations of canal surfaces, Bull. Korean Math. Soc. 53 (2016), No. 2, pp. 461-477.

[7] M.A. Soliman, W.M. Mahmoud, E.M. Solouma, and M. Bary. The new study of some characterization of canal surfaces with Weingarten and linear Weingarten types according to Bishop frame, J. of the Egyptian Math. Soci., (2019) 27-26.

[8] R A. Abdel-Baky. Developable surfaces through sweeping surfaces, Bull. of the Iranian Math. Society, 45(2019), p.p. 951-963.

[9] HY. Zhao, GJ. Wang. A new method for designing a developable surface utilizing the surface pencil through a given curve. Progress in Nature Science 2008; 18: 105-110.

[10] HB. Karadag, E. Kılıç, M. Karadag. On the developable ruled surfaces kinematically generated in Minkowski 3-space. Kuwait J. Sci. Eng. 41(1), 2012; 19-41.

[11] MT Aldossary \& RA. Abdel-Baky. On the Bertrand offsets for ruled and developable surfaces, Boll. Unione Mat. Ital., (2015), 8:53-64.

[12] RL. Bishop. There is more than one way to frame a curve. Amer. Math. Monthly 82, (1975), 246-251.

[13] F. Klok. Two moving coordinate frames for sweeping along a 3D trajectory. Comput. Aided Geom. Design 3, (1986), 217-229.

[14] W. Wang, and B. Joe. Robust computation of the rotation minimizing frame for sweep surface modelling. Computer-Aided Design (1997); 29:379-91.

[15] W. Wang, B. Jüttler, D. Zheng, andY. Liu. Computation of rotating minimizing frames. ACM Transactions on Graphics (2008); 27: 118.

[16] B. Bükcu and M. K. Karacan. On the slant helices according to Bishop frame of the timelike curve in Lorentzian space, Tamkang J. Math. 39(3) (2008), 255-262.
[17] O. Keskin and Y. Yayli. An application of N-Bishop frame to spherical images for direction curves, Int. J. Geom. Methods Mod. Phys. 14(11) (2017), 1750162 (21 pages).

[18] B. O'Neil. Semi-Riemannian Geometry geometry, with applications to relativity, Academic Press, New York, 1983.

[19] J. Walfare. Curves and Surfaces in Minkowski Space, Ph.D. Thesis, K.U. Leuven, Faculty of Science, Leuven, 1995.

\section{Contribution of individual authors to the creation of a scientific article (ghostwriting policy)}

Author Contributions: Please, indicate the role and the contribution of each author:

Example

John Smith, Donald Smith carried out the simulation and the optimization.

George Smith has implemented the Algorithm 1.1 and 1.2 in $\mathrm{C}++$.

Maria Ivanova has organized and executed the experiments of Section 4.

George Nikolov was responsible for the Statistics.

Follow: www.wseas.org/multimedia/contributorrole-instruction.pdf

\section{Sources of funding for research presented in a scientific article or scientific article itself} Report potential sources of funding if there is any

\section{Creative Commons Attribution License 4.0 (Attribution 4.0 International, CC BY 4.0)}

This article is published under the terms of the Creative Commons Attribution License 4.0 https://creativecommons.org/licenses/by/4.0/deed.en_US 